\title{
Outdoor colour recognition system for oil palm fresh fruit bunches (ffb)
}

\author{
Wan Ishak Wan Ismail ${ }^{1,2}$ and Mohd. Hudzari Razali ${ }^{1}$ \\ ${ }^{1}$ Intelligent System and Robotics Laboratory, Institute of Advanced Technology, Universiti Putra Malaysia \\ ${ }^{2}$ Department of Biological and Agriculture Engineering, Faculty of Engineering, Universiti Putra Malaysia, \\ 43400 UPM Serdang, Selangor, Malaysia, wiwi@eng.upm.edu.my
}

\begin{abstract}
The variations of day light intensity must be taken into account to recognize the color of the agriculture product when using camera vision system. In this study, the development of outdoor image analysis for oil palm fruit fresh bunches (FFB) was developed to analyses image of oil palm FFB. The software analysis will generates the mathematical model and correlation factor between the light intensity in relation to value of FFB from Red, Green and Blue component (RGB) of image taken. The visual basic programming language was used. An Excel worksheet programming software was developed to draw a chart and modify the charts location and dimensions. The API (Application Programming Interface) called GDI32 (Graphic Device Interface: 32-bit version) was applied to read and manipulate the pixel value of image. The software automation technique was used in the development of outdoor image analysis for agriculture product. The automation concept is to describe the automatic generation of the software to manipulate the data for producing the graph, mathematical analysis and equation, automatically from a bundle of data collected by only a click of mouse button. The result generated was used to develop mathematical model for color-based recognition for outdoor image of agriculture product. Linear regression function was used to find the equation of mathematical model for outdoors image analysis. To find the best equation of mathematical model, various trendline of data manipulation can be chosen manually from option provided in Microsoft Excel. The images of FFB were taken by using sony digital camera Handycam at various times of day from morning to afternoon in the oil palm plantation.
\end{abstract}

Keywords- Outdoors Vision System, Colour Recognition, Software Automation, Oil Palm FFB

\section{Introduction}

Vision is the most powerful sense. It provides us with a remarkable amount of information of our surroundings and enables us to interact intelligently with the environment. Vision is also one of the most complicated senses. With the application of modern technology, machines with a sense of vision are widely used in the industrial sector. In the industrial sector, vision systems are used under working environment. Vision systems are used to detect products, for quality control purposes by their shapes and patterns. Machine vision is a technology that employs a computer and camera to analyse and interpret images in a manner resembling human vision. The camera is equivalent to the human eye and the computer is equivalent to the human brain. Vision systems are a new field of research in the agricultural sector and the knowledge on how biological vision systems operate is directly concerned with signals from the sensors. In agricultural applications, especially for fruit handling, we cannot detect fruit quality just by its shape or pattern. This is because a fruit may have a different shape and pattern but the same level of quality. To solve this problem, the vision system should be able to analyse the colour of the object or fruit. In the harvesting and picking of oil palm fresh fruit bunches (FFB) in the field, a combination of shape, pattern and colour analysis in a vision system must be applied. The development of software for agriculture outdoors vision was successfully recognizes maturity of cocoa fruits by using colour recognition. The weakness of this concept is the user needs to update the reference color for fruit detection because of the changing of light intensity in outdoors environment [Wan Ishak et al, 2000]. The variations of the day light change the light intensity thus changing the RGB of the agriculture products. From the study review, it's possible to model the outdoor condition system to control the image color of the agricultural product. The trial outdoors image modelling was initially collected and shows that there is the linear changing on the pixel value of component RGB versus time (light intensity) as shown in Figure $(1 \mathrm{a}, 1 \mathrm{~b})$. The objective of this study is to model the system that can determine the target image at the outdoor environment with change light intensity. The changing of the light intensity of the environment will be measured using light meter. The fruit image will be captured simultaneously with day light intensity to model the relationship. This paper explains the development tools of automation that was use to capture, analyse and manipulate the image data of pixel value.

\section{Literature Review}

Machine vision applications for automated inspection and sorting of fruits and vegetables have been studied and reported; colour vision systems have been found more effective in colour inspection than monochrome systems. A colour camera output can be decoded into three images to represent the RGB components of the full image. The three components of the colour image can be recombined in software or hardware to produce intensity, saturation and hue images, which can be more convenient for subsequent processing. 
In computer, the intensity of the colours is based on bits. It is 24-bit colour (bits red, bits green and bits blue), each representing a nonnormalized RGB co-ordinate with a decimal value from 0 to 255 [Rafael and Richard, 2002]. In agro-based industries, researchers have used digital image analysis for bruised fruit detection. Wan Ishak et al. (1998) used colors analyse to grade 8 grades of 'pisang emas' banana. They found out that the 8 grades of banana which based on it degree of maturity, had own pixel value of RGB color component. Wan Ishak et. al (2000) also determined the RGB value of 6 categories of Oil Palm Fruit Bunches tested indoor environment condition. This project was conducted to determine and differentiate between the colour properties of oil palm fruit bunch. The camera vision system was able to detect and differentiate the ripe oil palm FFB from the other categories of oil palm FFB. A computer program developed was able to send a signal to the parallel port or printer port of the computer. The signal, in the form of electrical pulse, was used to turn on the LED that was 'attached to the port. The signal can be used to turn on a switch to activate the harvester. This initial study was only carried out in the laboratory environment under controlled conditions. The distances between the camera and the object and source of lights were made constant. Shashi (2002) develops models for illumination and surface reflectance for use in outdoor color vision, and in particular for predicting the color of surfaces under outdoor conditions. This development of a model of daylight that is more appropriate for outdoor machine vision applications, in that it (i) is indexed by imaging conditions and (ii) simulates a wide field of view. This makes it possible to predict the color of light incident on flat surfaces in outdoor scenes. There were, however, some limitations to this model. In particular, the date for the daylight model was sampled at three relatively similar geographical locations in North America; it is not clear if it applies to other parts of the world. Specifically, the effect of humidity, haze, and other particulate matter in the air may significantly affect the model. In addition, the daylight model does not account for the effect of light reflected from objects other than the ground. Nonetheless, it provides a method for approximating the color of daylight in real images. A series of experiments measure the accuracy of the daylight and reflectance models, and their applicability to outdoor color vision applications, by predicting the colors of surfaces in real images. The development of the "robot eye" system for cocoa harvester robot could recognize the target fruit on real plantation environment and can predict the actual distance of the object target (Hudzari, 2003). Stereo pair of Videogrammetry (finding the geometry using video) technique and triangulation was used and integrated. It is to determine the $X, Y$ and $Z$ axis distances measurement of object target. After the target thresholds was defined (as shown in Figure (2)) on the image as displayed on the graphical user interface (GUI), the three-dimensional target distance will be generated and robot arm able to move and grab the selected target automatically. The GUI was developed using visual basic version 6 programming languages. The development of mathematical model for robot arm was based on Denavit-Hertenberg kinematics solutions. The model was developed for the joint relationship of robot arm for producing the robot movement with real time simulation. Two digital cameras were used and fixed parallel to each other for applying the Videogrammetry concept to determine the coordinate detection. The user will trigger the signal from the computer to pneumatic solenoid valves of robot through controller card and produce proper output to locate robot arm. For the software automation concept, the Microsoft Office products (Word, Excel, PowerPoint, Access, and Outlook) provide servers that can be used to manipulate Office documents from Visual Basic or Visual Basic for Applications (VBA) code. Automation is a technology that allows Visual Basic programmers to rapidly incorporate the functionality of certain Windows-based application into the projects. Visual Basic program opens Excel program to initialise a series of cells, and uses the cells to display a chart to make software automation concept.

\section{Methodology}

The experiment started with capturing the image data and converts them to pixel value. It proceeds with migration data storage into Microsoft Excel program, graph formatting and presentation, embedded regression calculation and result of correlation value for mathematical modelling. This main graphical user interface was developed using Visual Basic programming language and linked to Microsoft excel using option via component reference. The outdoors image of the yellow flowers as in figure 1a was collected from 9:30am till 3:00pm to find the pixel value of red, green and blue component (RGB). Figure 3 below illustrates the software automation process chart. In making the software analysis development, the Windows Application Programming Interface (API) was used. The Windows API is a collection of dynamically linked libraries (socalled 'DLL files') that contain programming routines. These routines, or "interfaces" range from $\mathrm{I} / \mathrm{O}$ to networking to multimedia, and they generally work much faster than the intrinsic Visual Basic routines. The GDI32 functions are not an integral part of the Visual Basic programming language. To declare them in the General Declarations section just as we would a variable or a type [Michael, 1999]. The syntax of our API function declarations is as follows:

Private Declare Function SetPixelV Lib "GDI32" (ByVal hDC As Long, ByVal x As Long, ByVal y As Long, ByVal crColor As Long) As Byte 
- 'Private' simply means that we only want to use these functions within the current code block. If we were to use 'Public', we could use these function calls in any module, class module, or form in the current project.

- $\quad$ 'Lib "GDI32"' is short for 'Library "C:IWindows\System\GDI32.dll"' - it tells Visual Basic which dynamicallylinked library contains the code for the 'FunctionName' that was declared.

The values inside of the parentheses are nothing more than variable declarations.

- hDC: stands for "Handle Device Context." This is the computer's way of saying 'address of an object.' This variable tells us what picture box we want to draw on (like PictureBox1.hDC)

- $\quad X$ and $Y$ are the locations - in pixels of the pixel that want to work with. API calls always work in pixels. If the picture boxes use twips, inches, or any measurement other than pixels, these calls won't work.

- $\quad$ crColor is the color that we want to set pixel $(x, y)$ to.

One more API used in this project is the embedded reference used for automation process. 'Lib "EXCEL9.OLB"' is short for 'Library "C:IProgram Files\Mic..."' - it tells Visual Basic which dynamically linked library as shown in Figure (4). The automatic generator of data analysis and graph representation was embedded in dynamically linked library API. Figure (5a) shows the representative analysis result of the statistic. The automatic graph formatting as in Figure (5b) was done by applying the main source code as shown in Figure (5c) below. The colour of oil palm FFB was classified as black, hard, ripe, over ripe, empty bunch and rotten. This classification was taken from Kulumpang Development Corporation Sdn. Bhd. standards, which was based on PORIM (now known as MPOB) standards. The descriptions of the classifications were shown in Table 1. The samples were shown in Figure (6) and Figure (7) for Black and Hard Bunches respectively. A matured oil palm FFB, ready to be harvested, was categorized as 'ripe' fruit bunch. For the initial result in this project the sample of the yellow flower was tested and captured to find the RGB value from 9:30am till 2:00pm at 30 minuates interval. The development of Graphical User Interface was shown as in Figure (8). Figure 8 shows the image correlation efficient between pixel value versus difference of time. The samples of image of flower on outdoor condition was captured and 8 points was clicked on different image of various time. The small circle in Figure 8 indicate the area of point clicked on the images to get the pixel value of yellow flower. Figure (9) shows theregression analysis being carried to determine the correlation equation between the time and RGB pixel value. The detail linear regression result for outdoor image taken was shown in Figure (10).

\section{Result and Discussion}

The RGB component of a sample yellow flower were captured and analysed based on outdoor light condition. Figure 9 shows the result for pixel value for images taken and Table 2 below shows the correlation analysis as a measure of the reliability of the linear relationship between the $x$ and $y$ values. The red colour components show the highest correlation of 0.5688 followed by green and blue with a value of 0.1045 and 0.0832 . Tables 3,4 , and 5 show residual output of RGB colour component and the data line fit for RGB. All the data were obtained from regression result generated from excel as shown in Figure 10. For the data red, green and blue, the intercept value for a pixel values were $253.6786,250.9643,110.8571$ while the value for standard error are 6.902714, 7.8318, 22.40939 respectively. The blue colour component had the lowest pixel value with highest standard error. This is due to at the certain data captured show big error. The slope, which denote the time for red, green and blue colour components in this regression result were $-3.84524,-1.29762$ and -3.27381 respectively, and its standard error were $1.366941,1.550927$ and 4.43772 respectively. The red colour component had the highest slope with the highest differences of pixel value in period of time which also was found by Balasundram et al., (2007). All the value of slope had negative sign (-ve) to show that the value of RGB pixel was reduce in period of time. The graphical user interface software as shown in Figure 8 was developed using visual basic $V 6$ programming language that enhanced by API (application programming interface). The developed software was capable to measure the pixel value of selected points of yellow flower at various times and lighting intensity. These codes below are functions that will automatically extract the RGB pixel value of colour on every point clicked from computer screen:

Dim R as Byte, G as Byte, B as Byte

Dim Color as Long

Color $=$ PictureBox. Point $(0,0)$

$\mathrm{R}=$ ExtractR $($ Color $)$

$\mathrm{G}=$ ExtractG(Color)

$\mathrm{B}=$ ExtractB(Color)

The regression model calculation was successfully applied but the system needs the Analysis Toolpak kid add-in to be installed in Excel and the format data required need to select manually as shown in Figure (11a). The type of correlation for mathematical model can be chosen to get the best result that automatically generate onto computer screen as shown in Figure (11b). Figure (11c) shows the regression and correlation of the mathematical result. The linear regression type 
is normally used for this project. The software automation concept was successfully applied for tool development of outdoor image analysis. The automatic generation of the software can generate the Excel graph, equation result and analysis automatically from a bundle of image data by only a click of mouse button as shown in Figure $(8,9,10$ and 11). The pixel value of test image was successfully gathered and manipulated into separated component of red, green and blue (RGB pixel value) and the graph of pixel value versus time was plotted automatically like as shown in Figure (9) and Figure (11c). Two types of graphs will be drawn automatically;

- A residual plot will draw scatter plots of each independent variable on the $x$ axis, and the residual on the $y$-axis.

- A line fit plot will draw scatter plots of each independent variable on the $x$ axis, and the predicted and actual values of the dependent variable on the $y$-axis.

The correlation result of mathematical model will determine the best correlation coefficient. The function of correlation coefficient is to measure the reliability of the relationship between the $x$ (pixel value) and $y$ (times). Figure (9) show the correlation coefficient result for this project. The correlation coefficient closed to 1 indicated excellent linear reliability. Another mouse click was needed to get the regression result and correlation of mathematical modelling. Figure (10) and (11) shows the analysis of regression and correlation of mathematical result for test image taken.

\section{Conclusion}

This project was conducted to determine and differentiate between the pixel values of the object colour during the day in outdoor condition. The developed graphical analysis user interface was able to differentiate and give the result output of the RGB colour component differentiation. This initial study was only carried out on the yellow flower in outdoor condition of environment. The experiment should be conducted with the real Fresh Fruit Bunches (FFB) Oil Palm in the field for the real environment thus the categories of oil palm FFB maturity were determined. The analysis of this project is a step in considering the effect of the intensity of light, which changes with the time of the day. The factor of light intensity gives the major effect on outdoors image. The pixel value of colour component shows that intensity of light will change the pixel value of image. In addition the software automation concept is very important to develop the automatic software for outdoor image analysis. The complexity of designing and developing the agricultural vision are that they must be able to handle growing biological objects, withstand bad weather and other adverse conditions. The complexity also arises from the detection of agricultural products with a significant variability and complex biological environments, under in most cases, natural lighting conditions. The properties of growing plants and animals are dynamics and a lot of data are required to process automatically. The result of this study was to develop the mathematical colour modelling for outdoor image recognition that is the system can always determine the target image and solve the problem stated above.

\section{References}

[1] Kondo N. and Ting K.C. (1998) Robotics for Bioproduction System, ASAE, St. Joseph, Michigan, USA

[2] Wan Ishak W.I., Mohd Hudzari R., Khairuddin A.R., Muhammad Saufi Mohd M.K., Zakaria I., Mohd Fadhli I., Sharence A/L Nai Sowat. (2006) Sistem Cerdik Jentera Penyemburan Racun Rumpai, Malaysia Agriculture, Horticulture and Agrotourism Exhibition (MAHA 2006), Serdang, Selangor, Malaysia

[3] Rafael C. Gonzalez and Richard E. Woods (2002) Digital Image Processing, Prentice Hall, New Jersey

[4] Wan Ishak Wan Ismail and Lee Boon Huet (1998) First National Banana Seminar. Genting Highland Malaysia. Pg 148-156.

[5] Wan Ishak W. I., Mohd. Zohadie Bardaie, Abdul Malik Abdul Hamid (2000) Journal of Oil Palm Research 11(2),2, 38-45.

[6] Shasi Buluswar (2002) Models for Outdoor Color Vision, Doctoral dissertation, University of Massachusetts, Amherst

[7] Mohd. Hudzari Razali (2003) Videogrammetry Technique for Arm Positioning of BioProduction, Master Science Thesis, Universiti Putra Malaysia

[8] Michael H. (1999) Learn Microsoft Visual Basic 6.0 Now, Published by Microsoft Press, Washington

[9] Abdul Malik B. Hamid (1998) Camera Vision To Identify And Recognize The Colours Oil Palm Fruit Bunch, Final year thesis Universiti Putra Malaysia

[10] Harrell, R.C, Adsit P.D., Slaughter D.C. (1985) Real Time Vision -Servoing of a Robotic Tree-Fruit Harvester, ASAE Paper No. 853550. ASAE, ST. Joseph, Michigan, USA

[11] Idris O., Ashhar M. K., Haniff M.H. and Basri M. W. (2003) Color Meter for Measuring Fruit Ripeness, MPOB Information Series (195), ISSN 1511-7871.

[12] Abdullah M.Z., Guan L.C. and Mohd Azemi B.M.N. (2001) Stepwise Discriminant Analysis for Color Grading of Oil Palm using Machine Vision System, Institution of Chemical Engineers Trans Icheme, 79 Part C,223-231.

[13] Balasundram S.K., Robert P.C., Mulla D.J. (2006) Journal of Plant Sciences 1(3), 217-227.

[14] Yud R.C., Kuanglin C., Moon S.K. (2002) Computers and Electronics in Agriculture, 36, 173-191.

[15] Mohd. Hudzari Razali and Wan Ishak Wan Ismail (2007) World Engineering Congress (Wec07), Penang, Malaysia 


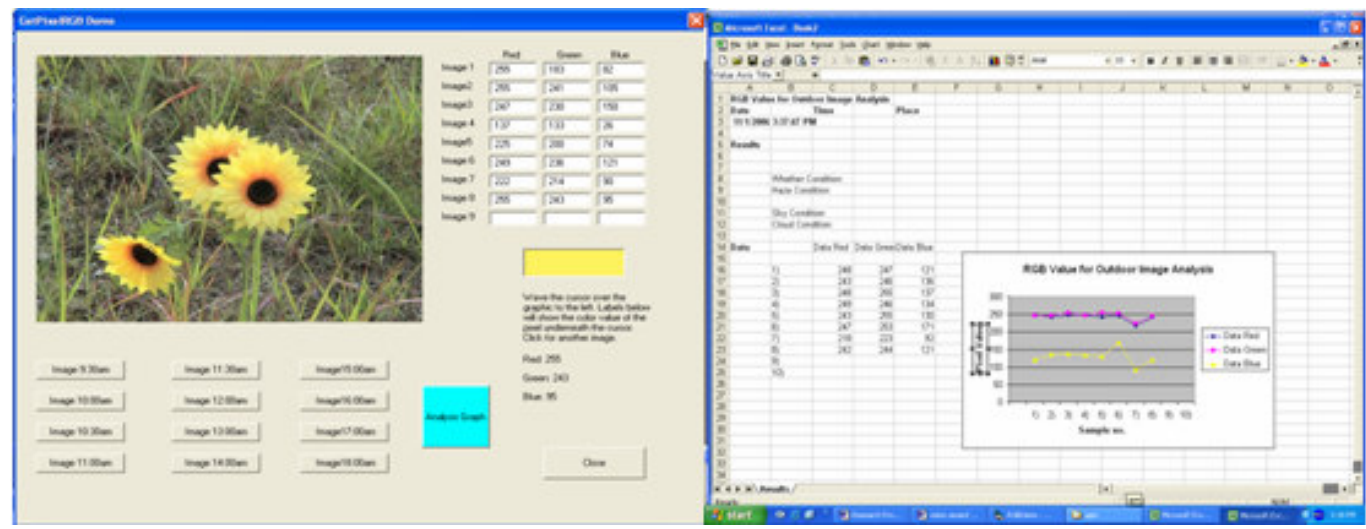

Fig. 1a, b- Developed software for color modelling analysis and graph relationship of pixel value of component rgb versus time (light intensity)

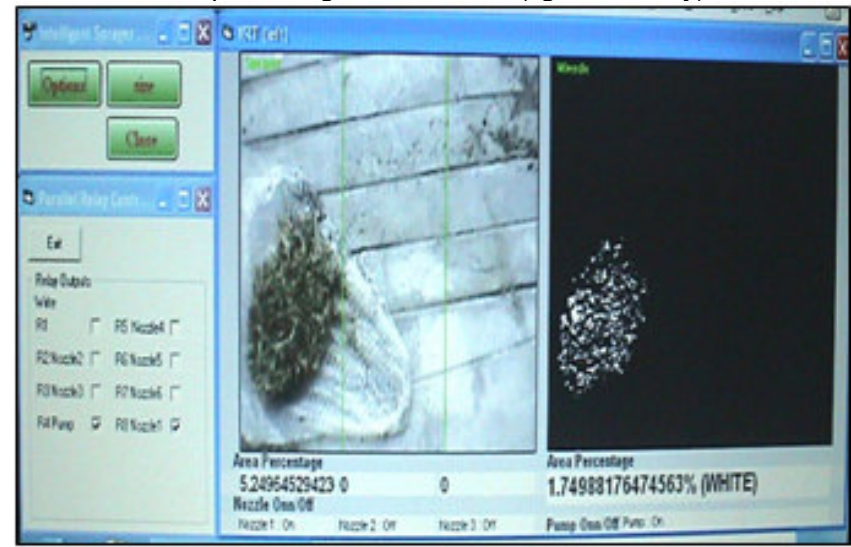

Fig. 2- Graphical user interface for outdoor vision (adapted from Wan Ishak et. al. (2006)

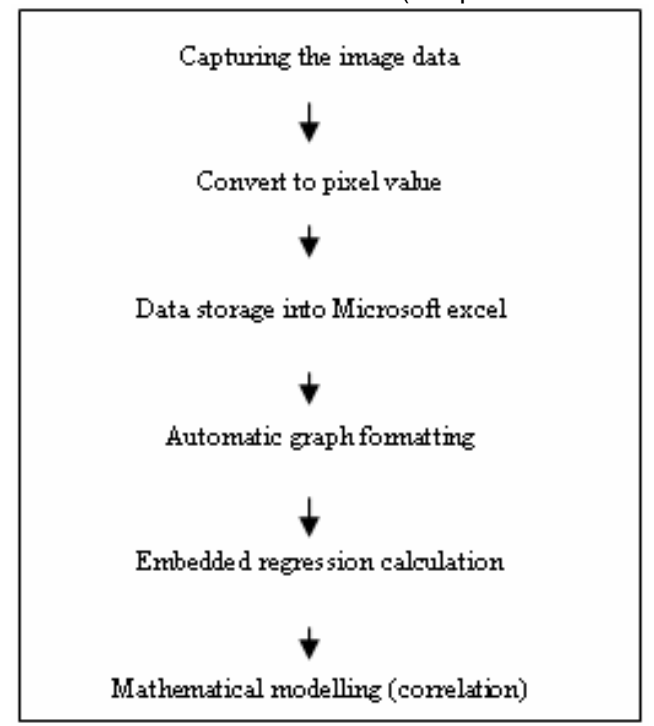

Fig. 3- Software automation process chart 


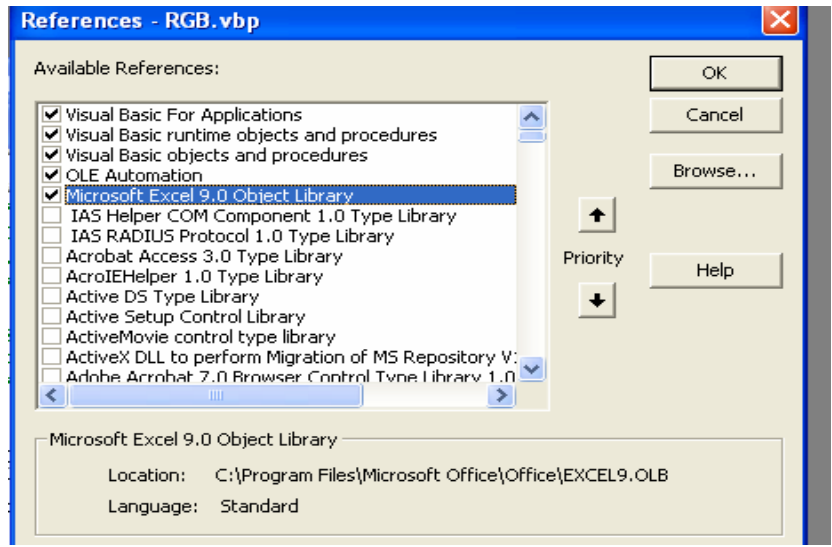

Fig. 4- Calling the Microsoft Excel Library from Visual Basic programming
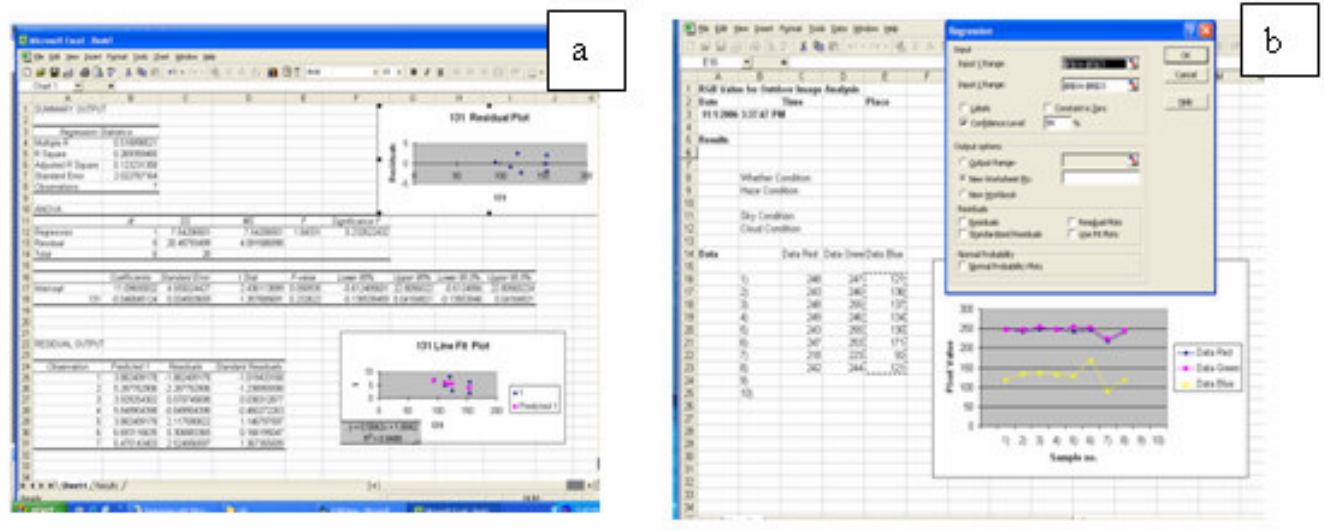

Charts.Add

ActiveChart. ChartType $=$ xlLineMarkers

ActiveChart.SetSourceData Source : Sheets ("Results") Range ("B14:E34"), PlotBy _

$:=x 1$ Columns

ActiveChart. Location Where: =xlLocationAsObject, Name: ="Results"

With ActiveChart

. HasTitle $=$ True

.ChartT itle.Characters. Text $=$ objWorksheet.Cells $(1,1)^{\prime}$ T itle

Axes (x1Category, x1Primary). HasTitle = True

Axes (x1Category, x1Primary).AxisTitle.Characters.Text = "Sample no." ' X-Axis

Axes (x1V alue, x1Primary) $\cdot$ HasTitle $=$ True

Axes (x1V alue, x1Primary).AxisTitle.Characters.Text = "Pixe1 V alue" \&

objWorksheet.Cells $(15,3)$ ' Y-Axis

End With

ActiveSheet.ChartObjects("Chart 1").Activate

With ActiveSheet.Shapes("Chart 1")

Left $=270.75$

Top $=178.5$

End With

Fig. 5a,b- The data analysis and graph generated from Visual Basic linked Microsoft Excel 


\begin{tabular}{|c|c|}
\hline Category & Description \\
\hline Black & Bunch with complete fruits \\
\hline Hard & Bunch with 1 to 9 fruits detached \\
\hline Ripe & $\begin{array}{l}\text { Bunch with } 10 \% \text { to } 50 \% \text { fruits } \\
\text { detached }\end{array}$ \\
\hline Over ripe & $\begin{array}{l}\text { Bunch with } 50 \% \text { to } 90 \% \text { fruits } \\
\text { detached }\end{array}$ \\
\hline Empty bunch & $\begin{array}{l}\text { Bunch with more than } 90 \% \text { fruits } \\
\text { detached }\end{array}$ \\
\hline Rotten & $\begin{array}{l}\text { Bunch with all or part having } \\
\text { turned black with or without fun- } \\
\text { gal attack }\end{array}$ \\
\hline
\end{tabular}

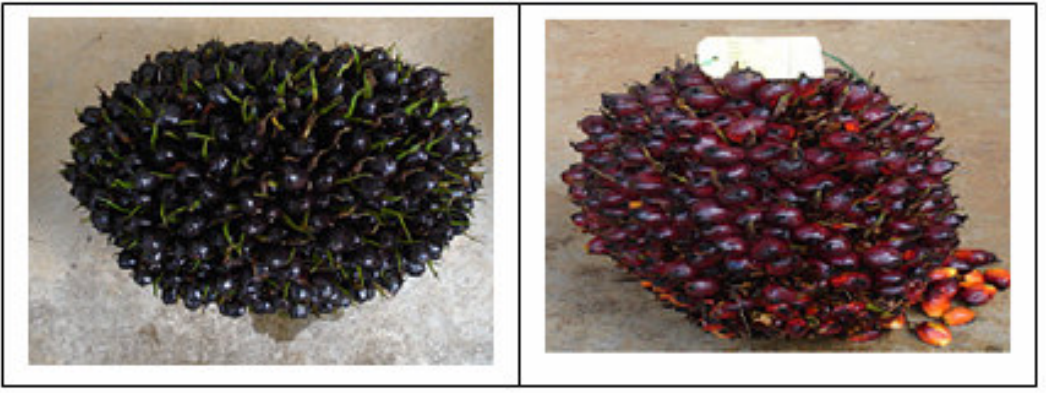

Fig. 6- Black Bunch

Fig. 7- Hard Bunch

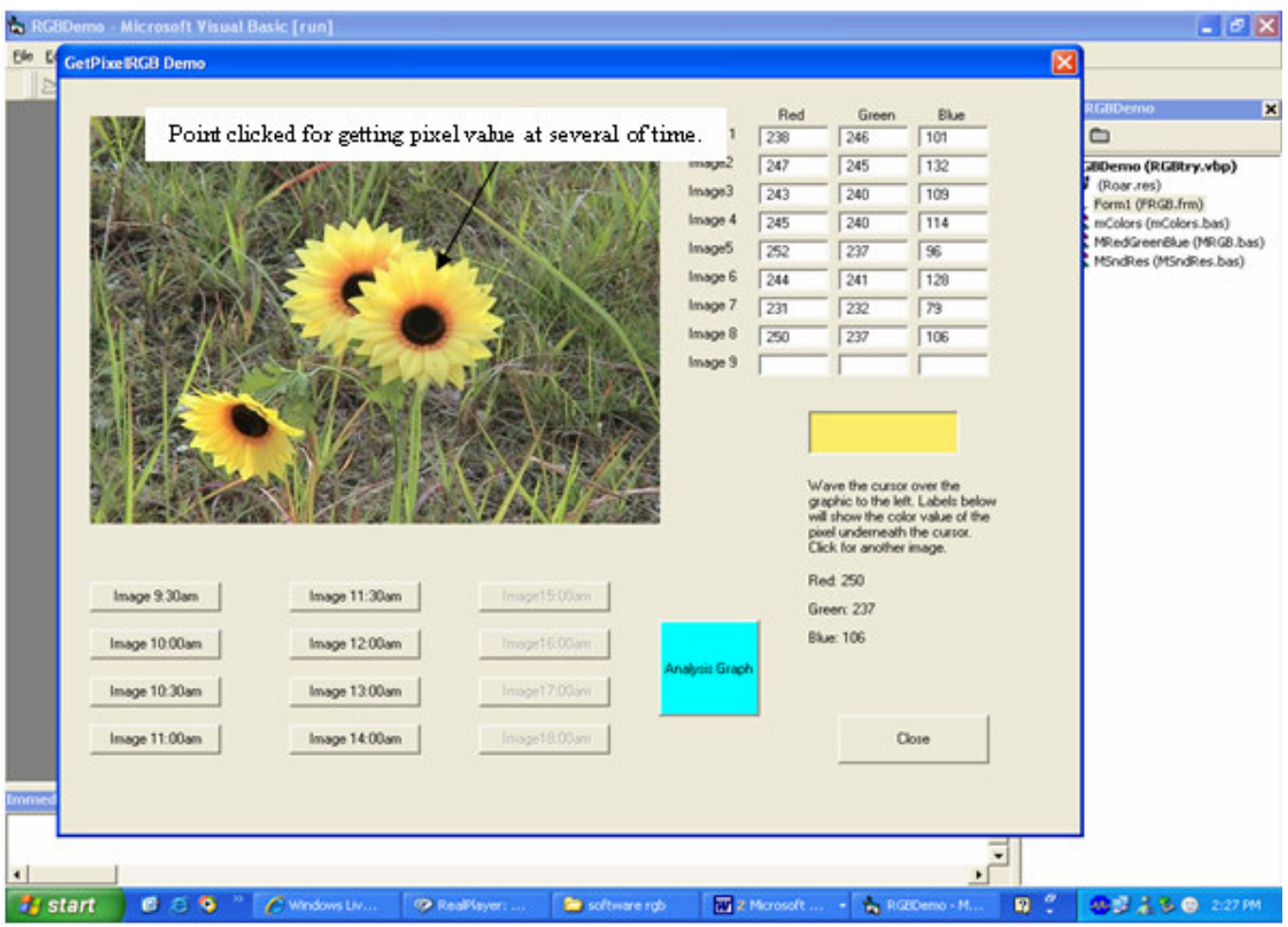

Fig. 8- Image correlation of yellow flowers between pixel value versus difference of time 


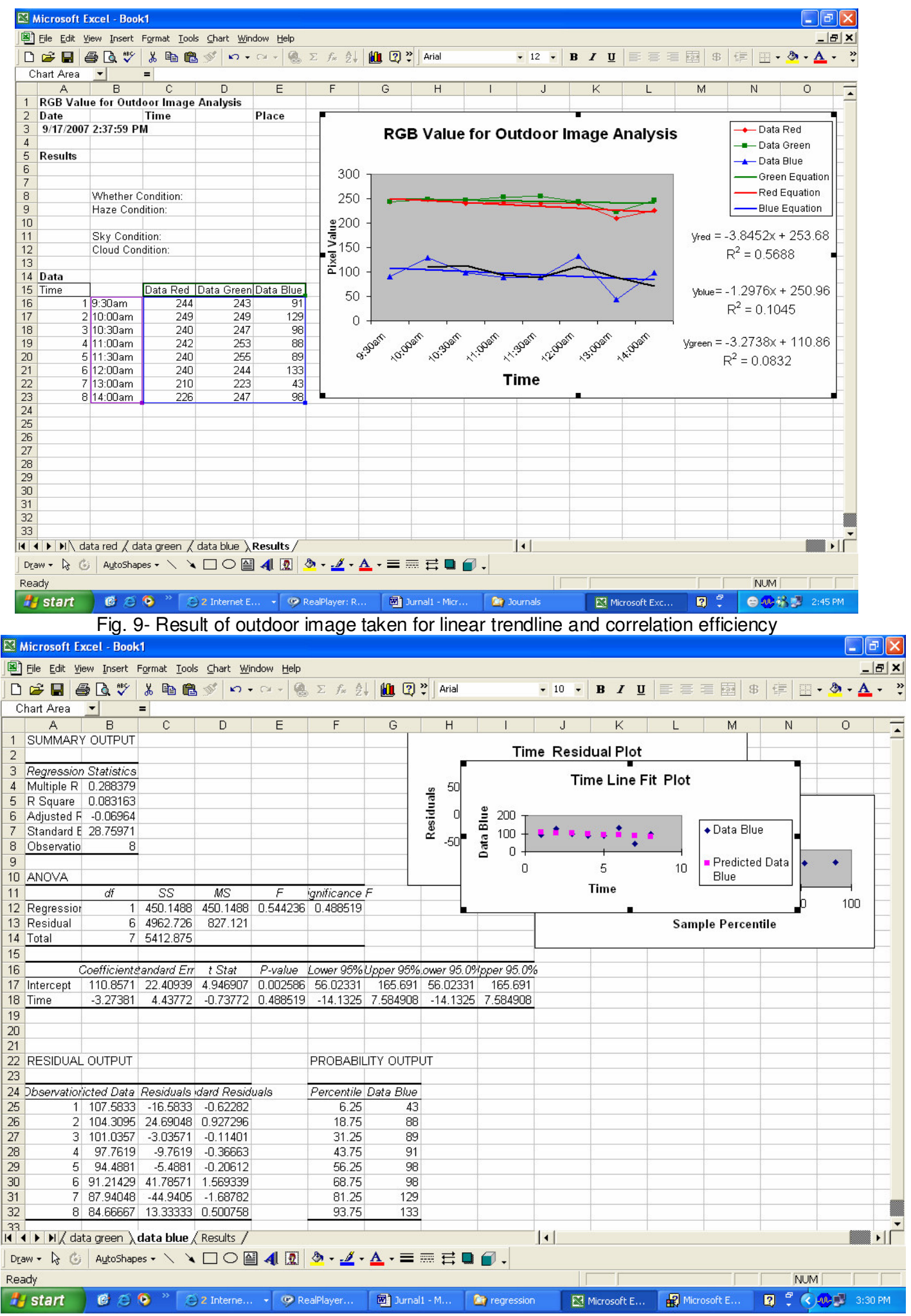

Fig. 10- Regression result with residual and line fit plot results 
Table 2- Correlation analysis of colour components

\begin{tabular}{|c|c|}
\hline $\begin{array}{c}\text { Colour } \\
\text { Component }\end{array}$ & Linear Equation \\
\hline Red & Yred $=-3.8452 \mathrm{x}+253.68$ \\
\hline Rreen & Yblue $=-1.2976 \mathrm{x}+250.96$ \\
\hline & $\mathrm{R} 2=0.1045$ \\
\hline Blue & Ygreen $=-3.2738 \mathrm{x}+110.86$ \\
\hline & $\mathrm{R} 2=0.0832$ \\
\hline
\end{tabular}

Table 3- The regression output for red colour component

\begin{tabular}{|c|c|c|c|}
\hline Observation & $\begin{array}{c}\text { Predicted } \\
\text { Data Red }\end{array}$ & Residuals & $\begin{array}{c}\text { Standard } \\
\text { Residuals }\end{array}$ \\
\hline 1 & 249.8333 & -5.83333 & -0.71114 \\
\hline 2 & 245.9881 & 3.011905 & 0.367232 \\
\hline 3 & 242.1429 & -2.14286 & -0.26117 \\
\hline 4 & 238.2976 & 3.702381 & 0.451419 \\
\hline 5 & 234.4524 & 5.547619 & 0.676403 \\
\hline 6 & 230.6071 & 9.392857 & 1.14524 \\
\hline 7 & 226.7619 & -16.7619 & -2.04372 \\
\hline 8 & 222.9167 & 3.083333 & 0.375941 \\
\hline & & & \\
\hline & Coefficients & Standard Error \\
\hline Intercept & 253.6786 & 6.902714 & \multicolumn{2}{|l}{} \\
\hline Time & -3.84524 & 1.366941 & \multicolumn{3}{|l}{} \\
\hline & & \multicolumn{3}{|l}{} \\
\hline
\end{tabular}

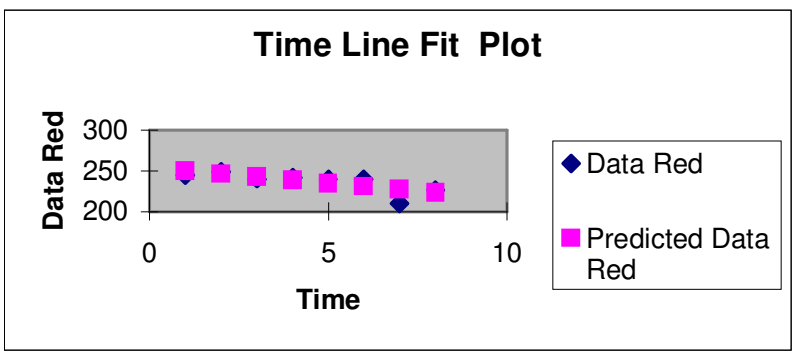

Table 4- The regression output for green colour component

\begin{tabular}{|c|c|c|c|}
\hline Observation & $\begin{array}{c}\text { Predicted } \\
\text { Data Green }\end{array}$ & Residuals & $\begin{array}{c}\text { Standard } \\
\text { Residuals }\end{array}$ \\
\hline 1 & 249.6667 & -6.66667 & -0.71642 \\
\hline 2 & 248.369 & 0.630952 & 0.067804 \\
\hline 3 & 247.0714 & -0.07143 & -0.00768 \\
\hline 4 & 245.7738 & 7.22619 & 0.776545 \\
\hline 5 & 244.4762 & 10.52381 & 1.130916 \\
\hline 6 & 243.1786 & 0.821429 & 0.088273 \\
\hline 7 & 241.881 & -18.881 & -2.029 \\
\hline 8 & 240.5833 & 6.416667 & 0.689552 \\
\hline & & & \\
\hline & Coefficients & Standard Error \\
\hline Intercept & 250.9643 & 7.8318 & \\
\hline Time & -1.29762 & 1.550927 & \multicolumn{3}{|l}{} \\
\hline
\end{tabular}




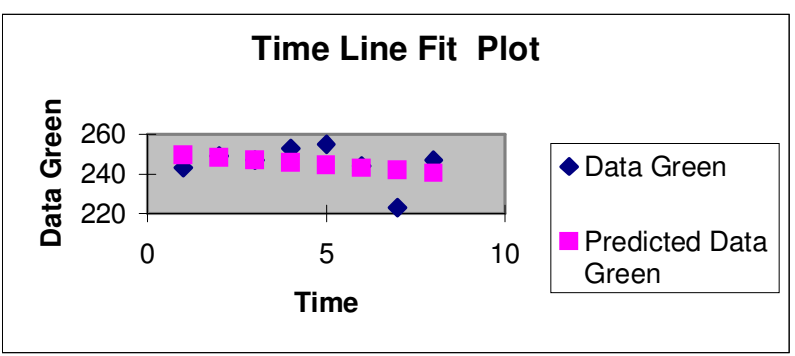

Table 5- The regression output for blue colour component

\begin{tabular}{|c|c|c|c|}
\hline Observation & $\begin{array}{c}\text { Predicted } \\
\text { Data Blue }\end{array}$ & Residuals & $\begin{array}{c}\text { Standard } \\
\text { Residuals }\end{array}$ \\
\hline 1 & 107.5833 & -16.5833 & -0.62282 \\
\hline 2 & 104.3095 & 24.69048 & 0.927296 \\
\hline 3 & 101.0357 & -3.03571 & -0.11401 \\
\hline 4 & 97.7619 & -9.7619 & -0.36663 \\
\hline 5 & 94.4881 & -5.4881 & -0.20611 \\
\hline & 91.21429 & 41.78571 & 1.569339 \\
\hline 7 & 87.94048 & -44.9405 & -1.68782 \\
\hline 8 & 84.66667 & 13.33333 & 0.500758 \\
\hline & & \multicolumn{3}{|c|}{} \\
\hline & Coefficients & Standard Error \\
\hline Intercept & 110.8571 & 22.40939 & \\
\hline Time & -3.27381 & 4.43772 & \multicolumn{3}{|l}{} \\
\hline
\end{tabular}
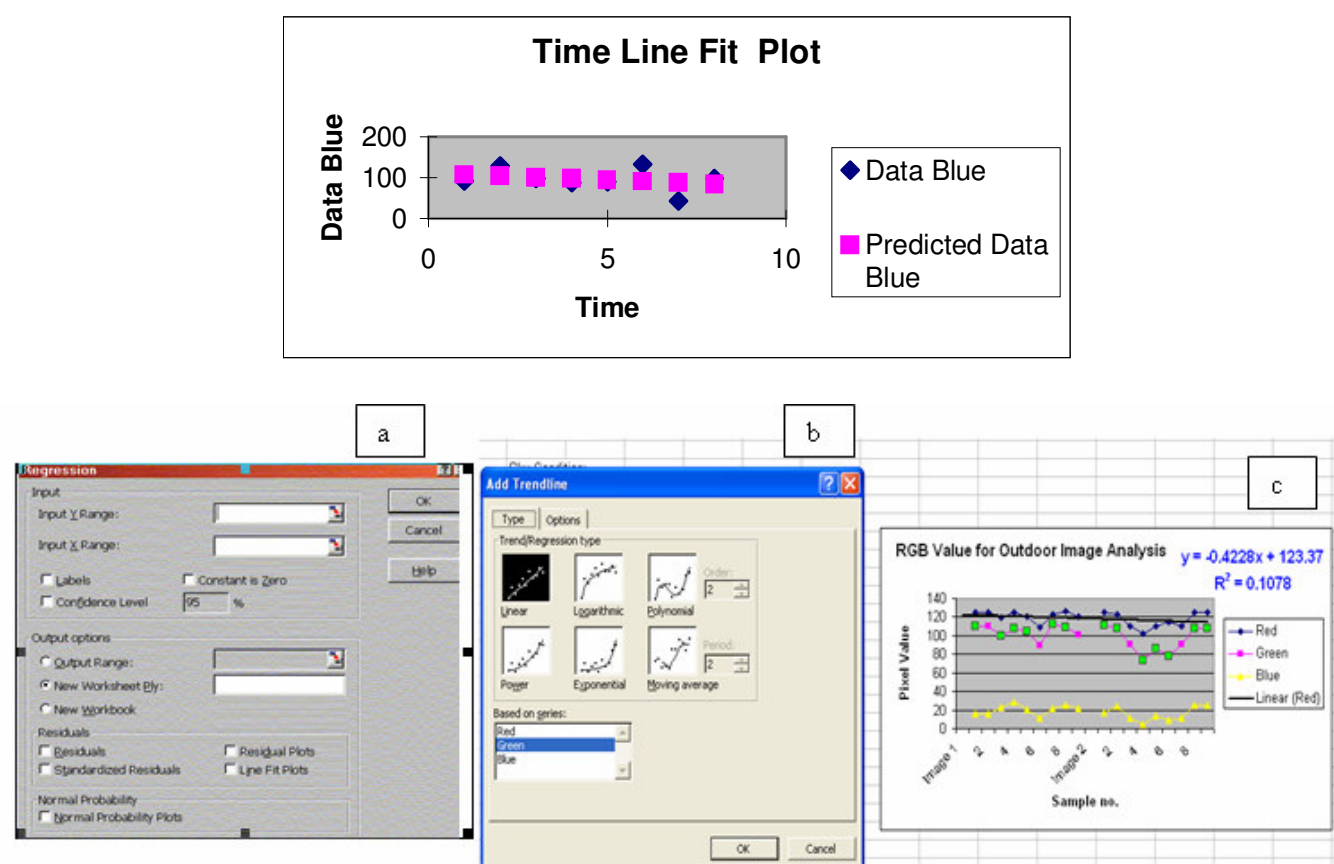

Fig. 11a,b,c- show toolbox and graph to produce the regression and correlation of mathematical result 